\title{
Energy Efficient Automized Botteling Plant Using Plc And Scada With Speed Variable Conveyor Assembly
}

\author{
Sagar P. Jain, Dr. Sanjay L. Haridas \\ M-Tech. scholar G.H.R.A.E.T., Nagpur \\ Dept. Of Electronics and Telecommunication Engg. G.H.R.C.E., Nagpur
}

\begin{abstract}
To accomplish we have applied knowledge of PLC and SCADA. A programmable logic controller (PLC) or programmable controller is a digital computer used for automation of electromechanical processes, such as control of machinery on factory assembly lines. Supervisory control and data acquisition refers to centralized systems which monitor and control entire sites. A variable-frequency drive (VFD) is used for controlling the speed of electric motor by controlling the frequency of the electrical power supplied to the motor. A fully automized bottling plant was made and the required efforts to achieve high efficiency and energy saving along with speed control was achieved.
\end{abstract}

\section{INTRODUCTION}

This research deals with process automation. It is necessary to have a system solution which can turn operational excellence onto a permanent condition, ensure that productivity and system work hand in hand as well create the foundation for plant flexibility. Earlier the processes carried out in industries were quite monotonous as a result the production could not be varied. The variation in production as per the requirement is a chief necessity in industries to survive the ever changing demand. The research offers a hardware assembly for a complete production line bottling facility incorporating the use of PLC and SCADA which in turn results into improved efficiency, energy saving and cost effectiveness.

The research deals with problems of controlling the speed of a conveyor belt for metal containers in a bottling plant. The primary objective of the controller is to guarantee continuous feed to the filling station, in presence of frequency gaps between bottles. The primary reason for using PLC is to eliminate the large cost involved over complicated Relay based machine control systems in order to overcome the production varying demands while providing a supervisory interface.

In many automation processes it is necessary to achieve a desired demand in some specified time. ExIf the production rate is 25 bottles per minute and the demand increases to 50 bottles per minute, the operating speed needs to be increased, whereas if the demand drops abruptly the production rate needs to be decreased. Thus the research deals with overcoming the problems of speed control in order to have improved operational parameters.

\section{PlC As System ConTroller}

A PLC is a microprocessor-based control system, designed for automation processes in industrial environments. It uses a programmable memory for the internal storage of user-orientated instructions for implementing specific functions such as arithmetic, counting, logic, sequencing, and timing. A PLC can be programmed to sense, activate, and control industrial equipment and, therefore, incorporates a number of I/O points, which allow electrical signals to be interfaced. Input devices and output devices of the process are connected to the PLC and the control program is entered into the PLC memory.

PLC's programming is based on the logic demands of input devices and the programs implemented are predominantly logical rather than numerical computational algorithms. Most of the programmed operations work on a straightforward two-state "on or off" basis and these alternate possibilities correspond to "true or false" (logical form) and "1 or 0" (binary form), respectively. Thus, PLCs offer a flexible programmable alternative to electrical circuit relay-based control systems built using analog devices. The programming method used is the ladder diagram method. The PLC system provides a design environment in the form of software tools running on a host computer terminal which allows ladder diagrams to be developed, verified, tested, and diagnosed. First, the high-level program is written in ladder diagrams. Then, the ladder diagram is converted into binary instruction codes so that they can be stored in random-access memory (RAM) or erasable programmable read-only memory (EPROM). Each successive instruction is decoded and executed by the CPU. The function of the CPU is to control the operation of memory and I/O devices and to process data according to the program. Each input and output connection point on a PLC has an address used to identify the I/O bit. The method for the direct representation of data associated with the inputs, outputs, and memory is based on the fact that the PLC memory is organized into three regions. 


\section{SCADA FOR MONITORING}

As implemented, RTUs and PLCs serve overlapping application niches and share some design details. To combat industry confusion, the discussion that follows provides a background of RTU and PLC units, and compares the various technical aspects for specifying the units including environmental ruggedness, modularity and scalability, and CPU performance. With remote applications for PLCs and RTUs continuing to expand, the discussion also helps the reader understand crucial remote system communication requirements including store and forward, report by exception, support for multiple protocols, and two-way communication with acknowledgements.

Many industrial and infrastructure-scale enterprises depend on equipment located at multiple sites dispersed over a large geographical area. A vast majority of large infrastructure and industrial-scale ventures use Supervisory Control and Data Acquisition (SCADA) systems.

SCADA systems provide monitoring, control, and automation functions that allow the enterprise to improve operational reliability, reduce costs through eased work force requirements, enhance overall Quality of Service (QoS), or meet expected QoS or other key performance factors as well as boost employee and customer safety.

\section{Block Diagram}

This deals with the key components used in settling up the complete plant and thus explains the use and working of each component. The block diagram of the experimental set up is illustrated. The following configurations can be obtained.

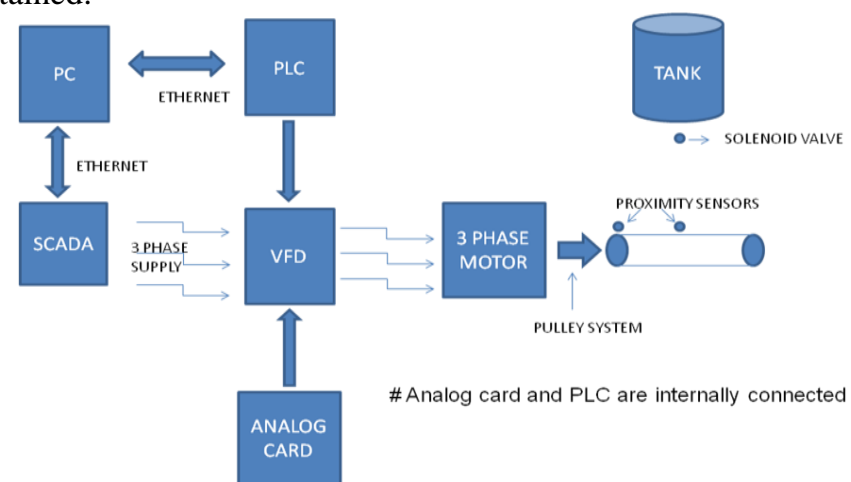

Figure 1: Block Diagram

The digital computer is used as an interface between PLC and SCADA. The PLC is a micro processor based system controller used to sense, activate and control industrial equipments and thus incorporate a number of input output/modules which allows electrical system to be interfaced. SCADA is a centralized system used to supervise a complete plant and basically consists of data accessing features and controlling processes remotely. The communication protocol used is Ethernet. The Variable Frequency Drive connected to the PLC receives AC power and converts it to an adjustable frequency adjustable voltage output for controlling the motor operation. The analog module converts analog input signals to digital output signals which can be manipulated by the processor.

The output of the VFD is given to the 3-phase induction motor which in turn with the help of a pulley mechanism is used to vary the speed of the conveyor belt.

An inductive sensor is an electronic proximity sensors used to detect metallic objects without touching them. The solenoid valve is a normally closed direct acting valve used to pour the liquid in the bottle whenever it gets a signal from the proximity sensor.

V.PLC AND RELATED SOFTWARE'S

The PLC used is MicroLogix 1400 as it has 20 inputs and 14 outputs and has an interface for Ethernet. The MicroLogix 1400 system offers higher I/O count, faster high-speed counter/PTO, and enhanced network capabilities The programming software used is RSLOGIX 500 and the communication software used is RS LINX 500.

Features of MicroLogix 1400:-

- Ethernet port provides Web server capability, email capability and protocol support

- Built-in LCD with backlight lets you view controller and I/O status

- Built-in LCD provides simple interface for messages, bit/integer monitoring and manipulation

- Expands application capabilities through support for as many as seven 1762 MicroLogix Expansion I/O modules with 256 discrete $\mathrm{I} / \mathrm{O}$

- As many as six embedded $100 \mathrm{kHz}$ high-speed counters (only on controllers with DC inputs)

- Two serial ports with DF1, DH-485, Modbus RTU, DNP3 and ASCII protocol support

\section{* Solenoid valves}


The Solenoid valves are available with different Orifice dimensions. The valve used has a dimension of $1 / 4^{\text {th }}$ of an inch. The reason for using the valve is as it is cost effective. Greater orifice dimensions allow greater flow of outlet liquid.

\section{* Proximity Sensors}

Proximity Sensors are available in two types namely;

1) Inductive sensors

2) Capacitive Sensors

Inductive Sensors are cheaper and allow detection of metal objects whereas capacitive sensors are costly and allow detection of metal, plastic and glass objects as well.

\section{* Variable Frequency Drive}

The Variable frequency drive is used to control the speed of the motor and works on the principle of pulse width modulation thus enabling speed control .The VFD used is POWERFLEX 4.

\section{Features}

- Power Ratings

- $100 \ldots 120 \mathrm{~V}: 0.2 \ldots 1.1 \mathrm{~kW} / 0.25 \ldots 1.5 \mathrm{Hp} / 1.5 \ldots 6 \mathrm{~A}$

- $\quad 200 \ldots 240 \mathrm{~V}: 0.2 \ldots 3.7 \mathrm{~kW} / 0.25 \ldots 5 \mathrm{Hp} / 1.4 \ldots 17.5 \mathrm{~A}$

- $\quad 80 \ldots 480 \mathrm{~V}: 0.4 \ldots 3.7 \mathrm{~kW} / 0.5 \ldots 5 \mathrm{Hp} / 1.4 \ldots 8.7 \mathrm{~A}$

- Ambient temperatures up to $50{ }^{\circ} \mathrm{C}$ permitted with minimal spacing between drives

- V/Hz control, slip compensation

- Internal RS-485 communications for communication with peripherals

- Drive overload protection, ramp regulation, and flying start

- Configuration and programming via integral LCD keypad, Drive Tool software.

\section{* 3-Phase Induction Motor}

In this reaserch speed control for various motors can be performed but the motor used is 3-phase induction motor, as it is cheaper and has gives good efficiency.

\section{* Methodology}

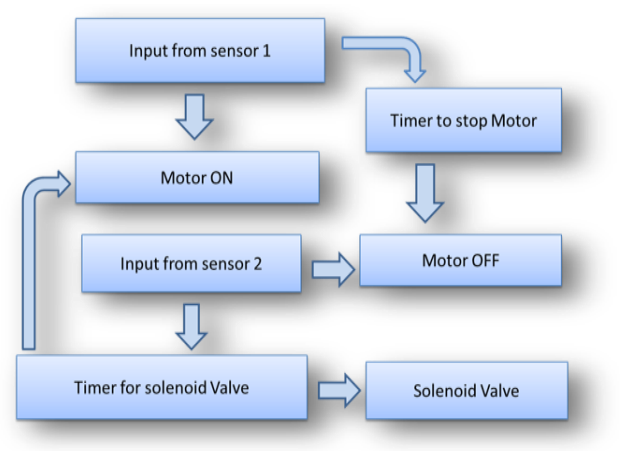

Figure 2 The flow of control signals to automate the process of bottle filling

To start the process of bottle filling, a bottle is placed on the conveyor belt in front of the first sensor. When sensor 1 senses the bottle, it gives a high output. This high bit is given to the PLC. The PLC in turn gives a signal to the inverter to start the 3 phase induction motor. The motor runs at a preset speed. The bottle now moves on the conveyor belt. The motor continues to run even when the bottle moves away from the first sensors range, i.e. the output of the motor is latched as explained in the ladder logic section of PLC.

When sensor 2 senses the bottle, it also gives a high output to the PLC. The PLC instructs the inverter to stop the motor. The high output bit of sensor 2 is also given to the timer for the solenoid valve. The timer used is $\mathrm{T}_{\mathrm{ON}}$. It counts for a predefined value of time $(18 \mathrm{sec})$. It gives two outputs, Enable output and done output. The Enable output remains high while the timer is counting and the output goes high after the timer has finished counting. The Enable output of $\mathrm{T}_{\mathrm{ON}}$ is given to the solenoid valve, and so the solenoid valve is open for the predefined value of time $(18 \mathrm{sec})$. The Done output bit is used to turn ON the motor again in the running condition.

If a condition arises that the bottle moving on the conveyor belt falls and is not able reach the sensor 2 , the conveyor will remain running and there will be unnecessary wastage of power. To overcome this problem, the high output bit of sensor 1 (normally open) is given in series with the output of sensor 2 (normally closed) to 
a timer. This timer counts for a waiting period of duration $10 \mathrm{sec}$. if the second sensors output does not go high within this period; the Done bit of the timer is used to stop the motor.

Another condition can be that some unauthorized person tries to fill the liquid by directly placing the bottle under the solenoid valve i.e. in front of sensor 2. The output of sensor will go high but the solenoid valve will not open. Condition has been set for the process that solenoid valve will open only and only if the motor is in the running condition.

\section{* Speed Control}

This research has the provision for making changes in the speed of the conveyor belt while the process is running. When the process is in the running condition, it is monitored by the SCADA. As shown in the window, there is a tag \# Drives freq. This tag allows the system supervisor to edit the speed of the conveyor belt, simply by double-clicking the \# Drive Freq tag and feeding the new value.

This new value in the tag is given to the analog card. This input signal to the analog card is converted into integer form. The PLC receives this integer signal and scales it. As shown in the screenshot below, the input bit to the Scale Control Parameter of the PLC is N7 which is taken from the analog card.

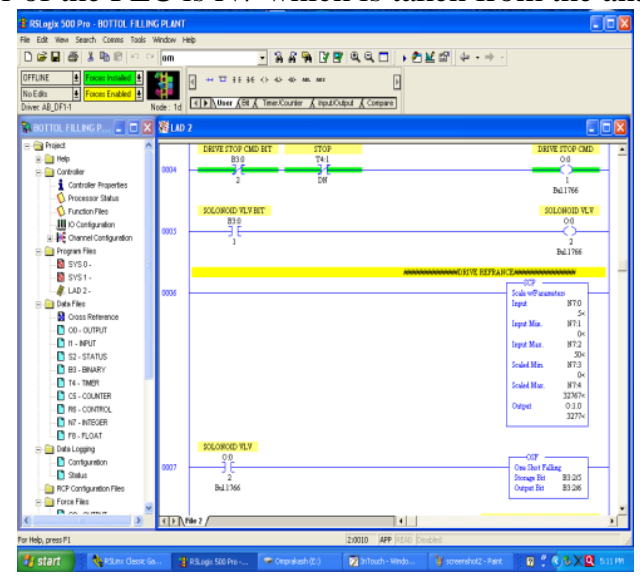

Figure 3 SCP Parameter

In the SCP, the minimum and maximum parameter according to which the input signal is to be scaled is predefined in the PLC. The SCP scales the signal from the analog card. The output signal from the SCP is again given to the analog card. The analog card acts as a D/A converter and generates a reference signal for the inverter in accordance to the received scaled signal. The inverter is a VFD which works on the principle of PWM. The reference signal from the analog card is used to generate a frequency. This new frequency is given to the motor. Since the speed of the motor is s function of frequency and is given by the formula $S=(120 \mathrm{f}) / \mathrm{P}$. Hence, the speed of the motor is changed, while the motor is still running.

\section{* Screenshots of SCADA}

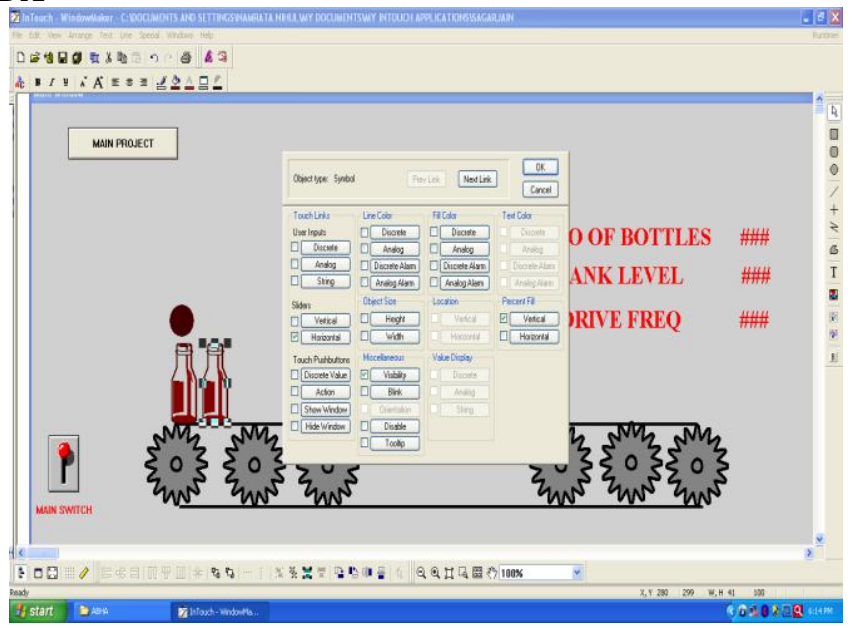

Figure 4: SCADA Run Time Window 


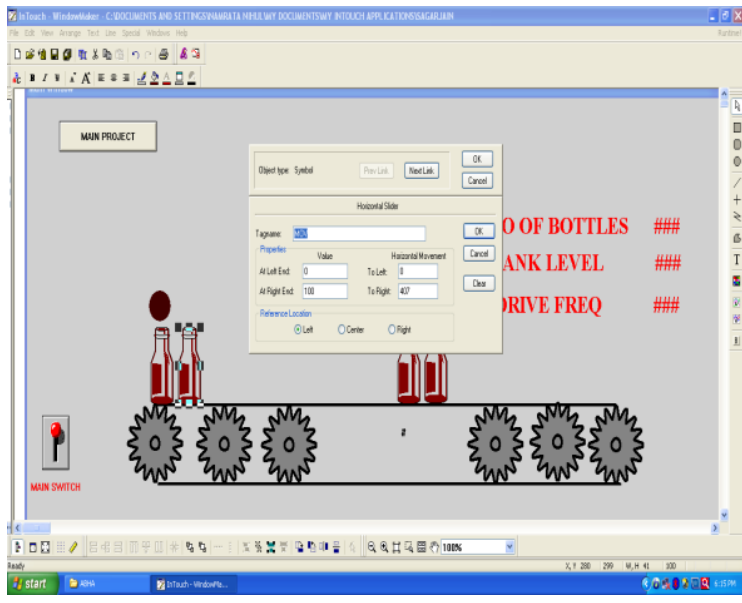

Figure 5: SCADA parameter Setting

* The Advantages and Disadvantages OF THE PLC AND SCADA SYSTEM

The advantages of the PLC and SCADA system are:

- The computer can record and store a very large amount of data

- The data can be displayed in any way the user requires

- Thousands of sensors over a wide area can be connected to the system

- The operator can incorporate real data simulations into the system

- Many types of data can be collected from the RTUs

- The data can be viewed from anywhere, not just on site

The disadvantages are:

- The system is more complicated than the sensor to panel type.

- Different operating skills are required, such as system analysts and programmer

- With thousands of sensors there is still a lot of wire to deal with

- The operator can see only as far as the PLC

\section{* Applications of PLC:}

Manufacturing Industry

- Lead acid battery plant, complete manufacturing system

- Extruder factory, silo feeding control systemTravel Industry

- Escalator operation, monitored safety control system

- Lift operation, monitored safety control systemAerospace

- Water tank quenching system

Printing Industry

- Offset web press print register control system

- Multistage screen washing system

Food Industry

- Filling machine control system

- Main factory feed water pump duty changeover system

Textile Industry

- Industrial batch washing machine control system

- Closed loop textile shrinkage system

Hospitals

- Coal fired boiler fan change-over system

Film Industry

- Servo axis controlled camera positioning system

Corrugating

- Main corrugation machine control system

- BOBST drive and control system

Plastics Industry

- Extruder factory, silo feeding control system

- Injection moulding control system 


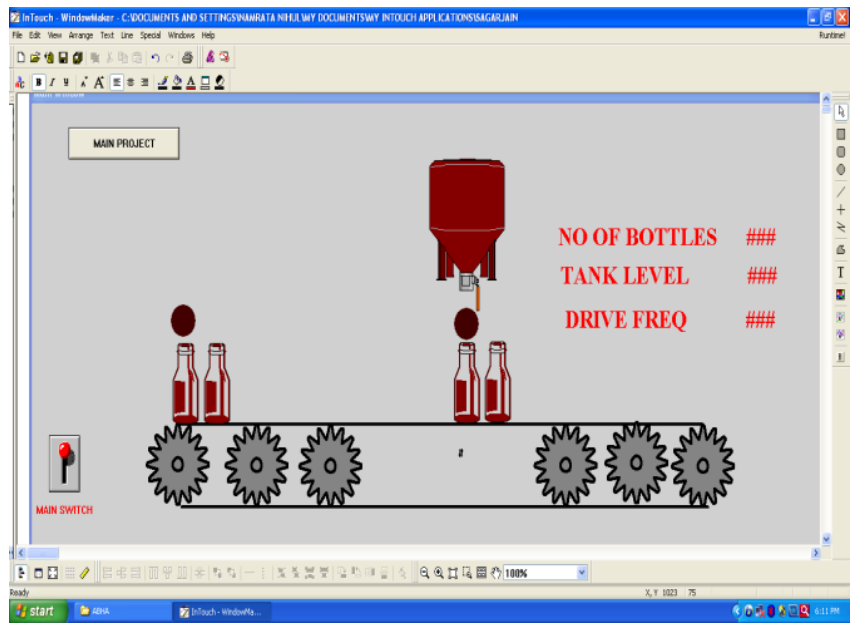

Figure 6: SCADA Tagging

\section{CONCLUSION}

In this paper conclude that the over all process offers a hardware assembly for a complete production line bottling facility incorporating the use of PLC and SCADA which in turn results into improved efficiency, energy saving and cost effectiveness. It is also overcoming the problems of speed control in order to have improved operational parameters.

\section{REFERENCES}

[1] G. Kaplan, "Technology 1992. Industrial electronics, IEEE Spect, vol.29, pp. 47-48, Jan. 1992.

[2] “Technology 1993. Industrial electronics,” IEEE Spectr. , vol. 30,pp. 58-60, Jan. 1993.

[3] A. R. Al-Ali, M. M. Negm, and M. Kassas, "A PLC based Power factor controller for a 3-phase induction motor," in Proc. Conf. Rec. IEEE Industry Applications, vol. 2, 2000, pp. 1065-1072.

[4] A. Hossain and S. M. Suyut, "Monitoring and controlling of a real time industrial process using dynamic model control technology," in Proc. IEEE Ind. Applicat. Soc. Workshop on Dynamic Modeling Control Applications for Industry, 1997, pp. 20-25.

[5] K. T. Erickson, "Programmable logic controllers," IEEE Potentials, vol.15, pp. 14-17, Feb/ Mar. 1996.

[6] B. Maaref, S. Nasri, and P. Sicard, "Communication system for industrial automation," in Proc. IEEE Int. Symp. Industrial Electronics, vol.3, 1997, pp. 1286-1291.

[7] Mader and H. Wuper, "Timed automation models for simple programmable logic controllers," in Proc. 11th Euromicro Conf. RealTime Systems, 1999, pp. 106-113.

[8] J. Marcos, E. Mandado, and C. M. Penalver, "Implementation of fail-safe control systems using programmable logic controllers," in Proc. IEEE/IAS Int. Conf. Industrial Automation and Control, 1995,pp. 395-400. 